\title{
The High Density Limit for Lattice Spin Models
}

\author{
Paul A. Pearce` and Colin J. Thompson \\ Mathematics Department, University of Melbourne, Parkville, Victoria 3052, Australia
}

\begin{abstract}
The $n$-vector, spherical and quantum spin models are considered on a regular lattice with co-ordination number $q$. In the limit $q \rightarrow \infty$ it is proved algebraically that the free energies are given by the corresponding Curie-Weiss or mean-field expressions.
\end{abstract}

\section{Introduction}

The classical Curie-Weiss theory of ferromagnetism has been established rigorously by considering various limiting lattice spin systems. It is known, for example (Thompson and Silver [1], Pearce and Thompson [2]), that if the spins interact with a Kac-type pair potential

$$
\varrho_{i j}=\gamma^{d} \varrho(\gamma|\boldsymbol{i}-\boldsymbol{j}|)
$$

the Curie-Weiss theory results in the long-range limit $\gamma \rightarrow 0$. In addition it has been shown (Pearce and Thompson [3]) that the Curie-Weiss theory arises for a system of $n$-vector spins, interacting with extreme anisotropy, in the spherical or infinite spin-dimensionality limit $(n \rightarrow \infty)$.

In this paper we will be concerned with spin systems on a regular lattice, with coordination number $q$, in the limit $q \rightarrow \infty$. This limit has been termed the high density limit by Brout [4] who first developed expansions for spin systems on lattices in inverse powers of the co-ordination number $q$, with the Curie-Weiss theory as leading term. More recently, Thompson [5] has proved that the $q \rightarrow \infty$ limit indeed results in the Curie-Weiss theory for Ising systems. However, the proof uses graphtheoretical methods and cannot be readily extended to other spin systems. Here, an algebraic method is developed that enables the treatment of both $n$-vector and quantum spin systems.

Consider $N$ spins occupying the sites of a $d$-dimensional lattice specified by the $d$-tuples

$$
i=\left(i_{1}, i_{2}, \ldots, i_{d}\right) \in \mathbb{Z}^{d}
$$

* Present address: Physics Department, Carnegie-Mellon University, Pittsburgh, PA 15213, USA 
with (not necessarily Cartesian) components

$$
i_{\mu}=1,2, \ldots, n_{\mu} ; \quad \mu=1,2, \ldots, d
$$

so that

$$
N=\prod_{\mu=1}^{d} n_{\mu} .
$$

Suppose further that the $\frac{1}{2} q N$ bonds $\langle\boldsymbol{i}, \boldsymbol{j}\rangle$ between interacting (typically nearestneighbour) pairs of spins forms a regular lattice graph (in the sense of Kasteleyn [6]).

Let the $n$-vector Hamiltonian be

$$
\mathscr{H}^{n}=-q^{-1} J \sum_{\langle i, j\rangle} S_{i} \cdot S_{j}-H \cdot \sum_{i} S_{i}
$$

where $J \geqq 0$ is the coupling constant, $\boldsymbol{H}$ is the external magnetic field and the $n$ dimensional spins have norm

$$
\left\|\boldsymbol{S}_{\boldsymbol{i}}\right\|=\left(\sum_{\mu=1}^{n} S_{\boldsymbol{i} \alpha}^{2}\right)^{1 / 2}=n^{1 / 2} .
$$

Then the $n$-vector free energy $\psi^{n}(\beta, \boldsymbol{H} ; q)$ is defined by $\left(\beta=1 / k_{B} T\right)$

$$
-\beta \psi^{n}(\beta, \boldsymbol{H} ; q)=\lim _{N \rightarrow \infty} N^{-1} \log Z_{N}^{n}(\beta, \boldsymbol{H} ; q)
$$

with

$$
Z_{N}^{n}(\beta, \boldsymbol{H} ; q)=A_{n}^{-N} \int_{\left\|\boldsymbol{S}_{j}\right\|=n^{1 / 2}} \ldots d^{N} \boldsymbol{S} \exp \left(-\beta \mathscr{H}^{n}\right)
$$

and

$$
A_{n}=2 \pi^{n / 2} n^{(n-1) / 2} / \Gamma(n / 2) .
$$

Similarly, let the quantum spin Hamiltonian be

$$
\mathscr{H}=-q^{-1} s^{-2} \sum_{\langle\boldsymbol{i}, \boldsymbol{j}\rangle} \boldsymbol{S}_{\boldsymbol{i}} \cdot \boldsymbol{J} \cdot \boldsymbol{S}_{\boldsymbol{j}}-s^{-1} \boldsymbol{H} \cdot \sum_{\boldsymbol{i}} \boldsymbol{S}_{\boldsymbol{i}}
$$

where, in the notation of [2], $S_{\boldsymbol{i}}$ is now the spin-s angular momentum operator for the spin at site $\boldsymbol{i}$ and $\boldsymbol{J}$ is a positive definite, symmetric anisotropy tensor. The quantum free energy $\psi(\beta, \boldsymbol{H} ; q)$ is then defined by

$$
-\beta \psi(\beta, \boldsymbol{H} ; q)=\lim _{N \rightarrow \infty} N^{-1} \log Z_{N}(\beta, \boldsymbol{H} ; q)
$$

with

$$
Z_{N}(\beta, H ; q)=\operatorname{Tr} \exp (-\beta \mathscr{H}) .
$$

The precise statement of our results is now as follows: 
Theorem 1. For the n-vector spin system specified by (1.5)-(1.9), the limiting free energy is given by

$$
\begin{aligned}
\psi^{n}(\beta, \boldsymbol{H}) & =\lim _{q \rightarrow \infty} \psi^{n}(\beta, \boldsymbol{H} ; q) \\
& =\min _{x}\left\{\frac{1}{2} n J x^{2}-\beta^{-1} \log \mathscr{I}_{n}(\beta J x+\beta H)\right\}
\end{aligned}
$$

where $H=n^{-1 / 2}\|\boldsymbol{H}\|$ and

$$
\mathscr{I}_{n}(x)=\Gamma(n / 2) I_{n / 2-1}(n x) /\left(\frac{1}{2} n x\right)^{n / 2-1},
$$

with $I_{\mu}$ the modified Bessel function of order $\mu$.

Theorem 2. For the quantum systems described by (1.10)-(1.12), the limiting free energy is given by

$$
\begin{aligned}
\psi(\beta, \boldsymbol{H}) & =\lim _{q \rightarrow \infty} \psi(\beta, \boldsymbol{H} ; q) \\
& =\min _{\boldsymbol{x}}\left\{\frac{1}{2} \boldsymbol{x} \cdot \boldsymbol{J} \cdot \boldsymbol{x}-\beta^{-1} \log C_{s}(\beta\|\boldsymbol{J} \cdot \boldsymbol{x}+\boldsymbol{H}\|)\right\}
\end{aligned}
$$

where

$$
C_{s}(s x)=\sinh \left(s+\frac{1}{2}\right) x / \sinh \frac{1}{2} x .
$$

The expressions (1.13) and (1.15) for the limiting free energies are precisely the Curie-Weiss or mean-field expressions. Notice also that the lattice-dimensionality $d$ does not appear explicitly in the statement of the theorems. We stress that $d$ need not be fixed; it is allowed to tend to infinity with $q$. Of particular interest here is the case of nearest-neighbour interactions on a Cartesian (hypercubic) lattice for which $q=2 d$. In this special case, of an infinite spatial-dimensionality limit, Gates and Thompson [7] have investigated the behaviour of the correlation functions for the scalar spin models and obtained the Ornstein Zernike formula.

The layout of rest of the paper is as follows. Theorem 1 is proved in Section 2. Then in Section 3 we discuss the spherical model and sketch the proof of Theorem 2.

\section{The $n$-Vector Model}

To obtain the limiting free energy (1.13) we find upper and lower bounds that coalesce in the limit $q \rightarrow \infty$. The upper bound on the free energy is immediate since the partition function $(1.8)$ is always $([1,5])$ bounded below by the Curie-Weiss partition function, regardless of potential or dimension. In the present context Jensen's inequality leads to

$$
Z_{N}^{n}(\beta, \boldsymbol{H} ; q) \geqq\left[\exp \left(-\frac{1}{2} n \beta J x^{2}\right) \mathscr{I}_{n}(\beta J x+\beta H)\right]^{N},
$$

provided $x$ is the magnetization given by

$$
x=\mathscr{F}_{n}(\beta J x+\beta H)
$$

where

$$
\mathscr{F}_{n}(x)=\mathscr{I}_{n}^{\prime}(x) / n \mathscr{I}_{n}(x)=I_{n / 2}(n x) / I_{n / 2-1}(n x) .
$$


Clearly, it follows that

$$
\psi^{n}(\beta, \boldsymbol{H} ; q) \leqq \min _{x}\left\{\frac{1}{2} n J x^{2}-\beta^{-1} \log \mathscr{I}_{n}(\beta J x+\beta H)\right\} .
$$

To obtain a lower bound on the free energy (1.13) we begin by writing the Hamiltonian (1.5) as

$$
\mathscr{H}^{n}=-\frac{1}{2} q^{-1} J \sum_{i, j} A_{i j} S_{i} \cdot S_{j}-H \cdot \sum_{i} S_{i}
$$

where

$$
A_{i j}=A_{j i}= \begin{cases}1 ; & i, j \text { interacting neighbours } \\ 0 ; & \text { otherwise }\end{cases}
$$

is the adjacency matrix. If we impose periodic boundary conditions the matrix $A$ becomes cyclic, i.e.

$$
A_{\boldsymbol{i} \boldsymbol{j}}=A(\boldsymbol{i}-\boldsymbol{j}), \quad A(\boldsymbol{k})=A(\boldsymbol{k}+\boldsymbol{n})
$$

where [cf. (1.4)]

$$
\boldsymbol{n}=\left(n_{1}, n_{2}, \ldots, n_{d}\right) \text {. }
$$

The matrix $q^{-1} A$ can now be diagonalized explicitly [8]. Defining

$$
S_{j k}=\prod_{\mu=1}^{d} n_{\mu}^{-1 / 2} e^{2 \pi i j_{\mu} k_{\mu} / n_{\mu}}
$$

we have

$$
S^{-1}\left(q^{-1} A\right) S=\operatorname{diag}\left(\lambda_{i}\right)
$$

where

$$
\lambda_{i}=\lambda\left(\frac{2 \pi i_{1}}{n_{1}}, \frac{2 \pi i_{2}}{n_{2}}, \ldots, \frac{2 \pi i_{d}}{n_{d}}\right)
$$

and

$$
\lambda(\boldsymbol{\theta})=\lambda\left(\theta_{1}, \theta_{2}, \ldots, \theta_{d}\right)=q^{-1} \sum_{\boldsymbol{k}} A(\boldsymbol{k}) e^{i \boldsymbol{k} \cdot \boldsymbol{\theta}} .
$$

To proceed with the derivation of the lower bound we wish to replace the matrix $q^{-1} A$ with a suitable positive definite matrix. To this end we define the cyclic matrix $K$ by

$$
K_{i \boldsymbol{j}}=q^{-1}|A|_{\boldsymbol{i} \boldsymbol{j}}+\varepsilon \delta_{\boldsymbol{i} \boldsymbol{j}} \quad(\varepsilon>0)
$$

where the non-negative definite matrix $|A|$ is given by

$$
q^{-1}|A|=S \operatorname{diag}\left(\left|\lambda_{i}\right|\right) S^{-1} .
$$

Since $|A|-A$ is non-negative definite (i.e. $A \leqq|A|)$ it follows immediately that $(v=\beta J$, $\boldsymbol{B}=\beta \boldsymbol{H})$

$$
Z_{N}^{n}(\beta, \boldsymbol{H} ; q) \leqq A_{n}^{-N} \int_{\left\|\boldsymbol{S}_{\boldsymbol{i}}\right\|=n^{1 / 2}} d^{N} \boldsymbol{S} \exp \left[\frac{1}{2} v \sum_{\boldsymbol{i}, \boldsymbol{j}} K_{\boldsymbol{i}} \boldsymbol{S}_{\boldsymbol{i}} \cdot \boldsymbol{S}_{\boldsymbol{j}}+\boldsymbol{B} \cdot \sum_{\boldsymbol{i}} \boldsymbol{S}_{\boldsymbol{i}}\right]
$$


Furthermore, since $K$ is positive definite, a standard identity [1] can be applied to the right hand side of $(2.15)$ to obtain

$$
\begin{gathered}
Z_{N}^{n}(\beta, \boldsymbol{H} ; q) \leqq(2 \pi)^{-N n / 2}\left(\operatorname{det} \frac{K}{v}\right)^{-n / 2} \int_{-\infty}^{\infty} \ldots \int^{N} d^{N} \exp \left(-\frac{1}{2} v \sum_{\boldsymbol{i}, \boldsymbol{j}} K_{\boldsymbol{i} \boldsymbol{j}}^{-1} \boldsymbol{x}_{\boldsymbol{i}} \cdot \boldsymbol{x}_{\boldsymbol{j}}\right) \\
A_{n}^{-N} \int_{\left\|\boldsymbol{S}_{\boldsymbol{i}}\right\|=n_{n^{1 / 2}} \ldots \int^{N} \boldsymbol{S} \exp \left[\sum_{\boldsymbol{i}}\left(v \boldsymbol{x}_{\boldsymbol{i}}+\boldsymbol{B}\right) \cdot \boldsymbol{S}_{\boldsymbol{i}}\right]}(2 \pi)^{-N n / 2}\left(\operatorname{det} \frac{K}{v}\right)^{-n / 2} \int_{-\infty}^{\infty} \ldots \int^{N} \boldsymbol{x} \exp \left[-\frac{1}{2} v \sum_{\boldsymbol{i}, \boldsymbol{j}}\left(K_{\boldsymbol{i} \boldsymbol{j}}^{-1}-z^{-1} \delta_{\boldsymbol{i} \boldsymbol{j}}\right) \boldsymbol{x}_{\boldsymbol{i}} \cdot \boldsymbol{x}_{\boldsymbol{j}}\right] \\
\prod_{\boldsymbol{i}} \exp \left[-\frac{1}{2} v z^{-1}\left\|\boldsymbol{x}_{\boldsymbol{i}}\right\|^{2}+\log \mathscr{I}_{n}\left(n^{-1 / 2}\left\|v \boldsymbol{x}_{\boldsymbol{i}}+\boldsymbol{B}\right\|\right)\right],
\end{gathered}
$$

where the function $\mathscr{I}_{n}$ arising from the configurational integrals (see [1]) is given by (1.14).

Maximising each term separately in the product occurring in (2.17), and performing the remaining Gaussian integrals we obtain our final bound $\left(x=n^{-1 / 2}\|\boldsymbol{x}\|\right)$

$$
\begin{aligned}
Z_{N}^{n}(\beta, \boldsymbol{H} ; q) \leqq & {\left[\operatorname{det}\left(I-\frac{K}{z}\right)\right]^{-n / 2} } \\
& \left\{\max _{x} \exp \left[-\frac{1}{2} n v z^{-1} x^{2}+\log \mathscr{I}_{n}\left(v x+n^{-1 / 2}\|\boldsymbol{B}\|\right)\right]\right\}^{N} .
\end{aligned}
$$

Obviously, this manipulation is valid only if the matrix $I-z^{-1} K$ is positive definite. To ensure this requirement we choose $z$ to be greater than the maximum eigenvalue of $K$, i.e. [see (2.13) and (2.11)], $z>1+\varepsilon$. This condition can be relaxed to $z>1$ if we let $\varepsilon \rightarrow 0+$. In this limit (2.18) still holds with the matrix $K$ replaced with the matrix $q^{-1}|A|$.

Since the matrix $|A|$ is a Toeplitz matrix, Szegö's theorem [9] gives

$$
\begin{aligned}
f(z ; q) & =\lim _{N \rightarrow \infty} N^{-1} \log \operatorname{det}\left(I-\frac{|A|}{q z}\right) \\
& =(2 \pi)^{-d} \int_{0}^{2 \pi} \ldots \int d \theta \log \left(1-\frac{|\lambda(\theta)|}{z}\right)
\end{aligned}
$$

where $\lambda(\theta)$ is given by (2.12). It follows then from (2.18) and (1.7) that

$$
\begin{aligned}
\psi^{n}(\beta, \boldsymbol{H} ; q) \geqq & \min _{x}\left\{\frac{1}{2} n z^{-1} J x^{2}-\beta^{-1} \log \mathscr{I}_{n}(\beta J x+\beta H)\right\} \\
& +\frac{1}{2} n \beta^{-1} f(z ; q)
\end{aligned}
$$

for all $z>1$. In view of the upper bound (2.4) we will have proved Theorem 1 , that is (1.13), once we have established that

$$
\lim _{z \rightarrow 1+} \lim _{q \rightarrow \infty} f(z ; q)=0 \text {. }
$$

This is proved in the Appendix.

\section{The Spherical and Quantum Spin Models}

The limiting free energy of the Berlin and Kac spherical model [10] can be obtained [11] from the result of the previous section. By considering $n^{-1} \psi_{n}(\beta, \boldsymbol{H} ; q)$ and 
taking the spherical limit $n \rightarrow \infty$ before (or after) the limits $q \rightarrow \infty, z \rightarrow 1+$ one obtains (see $[3,12]$ )

$$
\begin{aligned}
\psi_{\mathrm{sph}}(\beta, B) & =\lim _{n \rightarrow \infty} n^{-1} \psi_{n}(\beta, \boldsymbol{H}) \\
& =-\max _{0 \leqq x \leqq 1}\left\{\frac{1}{2} J x^{2}+B x+\frac{1}{2} \beta^{-1} \log (1-x)\right\},
\end{aligned}
$$

where

$$
B=\lim _{n \rightarrow \infty} n^{-1 / 2}\|\boldsymbol{H}\| .
$$

The expression (3.1) is precisely the free energy of the Curie-Weiss (or mean-field) spherical model.

Alternatively, the spherical free energy can be obtained directly by solving the spherical model on a regular lattice with co-ordination number $q$. In particular for zero field we have [13]

$$
-\beta \psi_{\mathrm{sph}}(\beta, 0 ; q)=\frac{1}{2}\left\{v z-1-\log v-(2 \pi)^{-d} \int_{0}^{2 \pi} \ldots \int d \boldsymbol{\theta} \log (z-\lambda(\boldsymbol{\theta}))\right\}
$$

with $\lambda(\theta)$ defined by (2.12) and $z$ determined by the saddle point condition

$$
v=(2 \pi)^{-d} \int_{0}^{2 \pi} \ldots \int_{0} \frac{d \theta}{z-\lambda(\theta)} .
$$

As $q \rightarrow \infty$ the right-hand side of (3.4) tends to $z^{-1}$ (see Lemma 2 in the Appendix), provided $z>1$. This is certainly the case when $v<1$. For $v>1$ the saddle point "sticks" at $z=1$ in three and higher dimensions $(d \geqq 3)$. In one and two dimensions, on the other hand, the spherical model does not have a phase transition for finite $q$ and the saddle point is given for large $q$ by

$$
z=1+o(1)
$$

when $v>1$. In both cases, from (3.3) we obtain in the limit $q \rightarrow \infty$,

$$
-\beta \psi_{\mathrm{sph}}(\beta, 0)= \begin{cases}0, & v<1 \\ \frac{1}{2}(v-1-\log v), & v>1\end{cases}
$$

in agreement with (3.1).

Theorem 2 for the quantum systems can be proved in essentially the same way as Theorem 1. The extra complications due to the non-commutation of the spin operators can be dealt with by the methods of [2]. The upper bound on the free energy, for example, is obtained by using Bogoliubov's variational principle. The derivation of the lower bound, however, is more involved. First Peierl's theorem is used to replace the matrix $q^{-1} A(2.6)$ with the matrix $K(2.13)$. Then Trotter's formula is used to obtain a functional integral representation. Following the method of [2] then gives a lower bound on the free energy of the required form [cf: (2.20)] with $\frac{3}{2} \beta^{-1} f(z ; q)$ [see (2.19)] as remainder. The most important thing to note is that, as in the $n$-vector case (see the Appendix), this remainder term vanishes in the limit $q \rightarrow \infty$. 


\section{Appendix}

Our aim here is to prove the result (2.21). This will be done in a series of Lemmas. Before proceeding it is convenient to introduce the lattice average

$$
\langle g(\boldsymbol{\theta})\rangle_{\boldsymbol{\theta}}=(2 \pi)^{-d} \int_{0}^{2 \pi} \ldots \int_{0} g(\boldsymbol{\theta}) d \theta_{1} d \theta_{2} \ldots d \theta_{d}
$$

and the inner product

$$
(f(\boldsymbol{\theta}), g(\boldsymbol{\theta}))=\left\langle f(\boldsymbol{\theta})^{*} g(\boldsymbol{\theta})\right\rangle_{\boldsymbol{\theta}} .
$$

It should be remarked here that the set

$$
\left\{e^{i \boldsymbol{k} \cdot \boldsymbol{\theta}}: \boldsymbol{k} \text { a lattice vector }\right\}
$$

is a complete orthonormal set of functions with respect to the inner product (A.2).

Lemma 1. If $A_{\boldsymbol{i} \boldsymbol{j}}=A(\boldsymbol{i}-\boldsymbol{j})$ is the adjacency matrix (2.6) for the regular d-dimensional lattice graph with co-ordination number $q$, and

$$
\lambda(\boldsymbol{\theta})=q^{-1} \sum_{\boldsymbol{k}} A(\boldsymbol{k}) e^{i \boldsymbol{k} \cdot \boldsymbol{\theta}}
$$

then

$$
\langle|\lambda(\boldsymbol{\theta})|\rangle_{\boldsymbol{\theta}} \rightarrow 0 \text { as } q \rightarrow \infty .
$$

Proof. By applying the Schwarz inequality and the Parseval identity we obtain:

$$
\begin{aligned}
0 \leqq\langle|\lambda(\boldsymbol{\theta})|\rangle_{\boldsymbol{\theta}} & \leqq\left\langle|\lambda(\boldsymbol{\theta})|^{2}\right\rangle_{\boldsymbol{\theta}}^{1 / 2}\langle 1\rangle_{\boldsymbol{\theta}}^{1 / 2} \\
& =\left\{\sum_{\boldsymbol{k}}\left|q^{-1} A(\boldsymbol{k})\right|^{2}\right\}^{1 / 2} \\
& =q^{-1 / 2} \rightarrow 0 \text { as } q \rightarrow \infty .
\end{aligned}
$$

Lemma 2. In the previous notation,

$$
\left\langle\frac{1}{z-|\lambda(\boldsymbol{\theta})|}\right\rangle_{\boldsymbol{\theta}} \rightarrow z^{-1} \text { as } q \rightarrow \infty,
$$

for all $z>1$.

Proof. Suppose $z>1$. Then by an elementary identity [6] and an integration by parts [observe that $|\lambda(\theta)| \leqq 1]$ we obtain:

$$
\begin{aligned}
z^{-1} \leqq\left\langle\frac{1}{z-|\lambda(\boldsymbol{\theta})|}\right\rangle_{\boldsymbol{\theta}} & =\int_{0}^{\infty} e^{-t z}\left\langle e^{t|\lambda(\boldsymbol{\theta})|}\right\rangle_{\boldsymbol{\theta}} d t \\
& =z^{-1}\left[1+\int_{0}^{\infty} e^{-t z}\left\langle|\lambda(\boldsymbol{\theta})| e^{t|\lambda(\boldsymbol{\theta})|}\right\rangle_{\boldsymbol{\theta}} d t\right] \\
& \leqq z^{-1}\left[1+\int_{0}^{\infty} e^{-t z}\langle|\lambda(\boldsymbol{\theta})|\rangle_{\boldsymbol{\theta}} e^{t} d t\right] \\
& =z^{-1}\left[1+(z-1)^{-1}\langle|\lambda(\boldsymbol{\theta})|\rangle_{\boldsymbol{\theta}}\right] \\
& \rightarrow z^{-1} \text { as } q \rightarrow \infty .
\end{aligned}
$$

In this last step we have used Lemma 1. 
Lemma 3. In the previous notation,

$$
\langle\log (z-|\lambda(\boldsymbol{\theta})|)\rangle_{\boldsymbol{\theta}} \rightarrow \log z \quad \text { as } \quad q \rightarrow \infty,
$$

for all $z>1$.

Proof. Suppose $z>1$. Then by Jensen's inequality and Lemma 1 we obtain:

$$
\begin{aligned}
\langle\log (z-|\lambda(\boldsymbol{\theta})|)\rangle_{\boldsymbol{\theta}} \leqq & \log \left(z-\langle|\lambda(\boldsymbol{\theta})|\rangle_{\boldsymbol{\theta}}\right) \\
& \rightarrow \log z \text { as } q \rightarrow \infty .
\end{aligned}
$$

But by Jensen's inequality and Lemma 2,

$$
\begin{aligned}
\langle\log (z-|\lambda(\boldsymbol{\theta})|)\rangle_{\boldsymbol{\theta}}= & -\left\langle\log \left(\frac{1}{z-|\lambda(\boldsymbol{\theta})|}\right)\right\rangle_{\boldsymbol{\theta}} \\
\geqq & -\log \left\langle\frac{1}{z-|\lambda(\boldsymbol{\theta})|}\right\rangle_{\boldsymbol{\theta}} \\
& \rightarrow \log z \text { as } q \rightarrow \infty .
\end{aligned}
$$

The result (A.7) follows. In particular, we have

$$
\begin{aligned}
f(z ; q)= & \langle\log (z-|\lambda(\boldsymbol{\theta})|)\rangle_{\boldsymbol{\theta}}-\log z \\
& \rightarrow 0 \text { as } \quad q \rightarrow \infty,
\end{aligned}
$$

for all $z>1$. The result (2.21) is now obtained by taking the limit $z \rightarrow 1+$.

\section{References}

1. Thompson, C.J., Silver, H. : Commun. math. Phys. 33, 53 (1973)

2. Pearce,P.A., Thompson, C.J.: Commun. math. Phys. 41, 191 (1975)

3. Pearce,P.A., Thompson, C.J.: Progr. Theoret. Phys. 55, 665 (1976)

4. Brout, R.: Phys. Rev. 118, 1009 (1960)

5. Thompson, C.J.: Commun. math. Phys. 36, 255 (1974)

6. Kasteleyn,P.W.: Graph theory and theoretical physics (ed. F. Harary), London: Academic Press 1967

7. Gates, D.J., Thompson, C.J.: J. Stat. Phys. 13, 219 (1975)

8. Montroll,E.W.: Applied combinatorial mathematics (ed. E. F. Beckenbach), New York : John Wiley 1964

9. Grenander,U., Szegö, G. : Toeplitz forms and their applications. Berkeley, California : University of California Press 1958

10. Berlin,T.H., Kac, M.: Phys. Rev. 86, 821 (1952)

11. Kac, M., Thompson, C.J.: Physica Norvegica 5, 163 (1971)

12. Pearce,P.A., Thompson, C.J.: J. Phys. A 10, 571 (1977)

13. Joyce, G.S.: Phase transitions and critical phenomena, Vol. 2 (ed. C. Domb, M. S. Green), London: Academic Press 1972

Communicated by E. Lieb

Received June 20, 1977 